\title{
THE ROLE OF BEAUTY IN PHYSICS
}

The goal of physics is to look for the truth behind the physical phenomena. Where is the place for the beauty then? The truth can be in principle ugly in one case and aesthetically pleasing in another. Nevertheless, many physical theories can be considered as beautiful and are often formulated with aesthetic criteria in mind. These criteria include the ability to explain a lot starting from a little and/or some form of symmetry. Several examples of beautiful laws will be discussed along with opinions of particle physicists on beauty.

Keywords: beauty in physical theories, symmetry, simplicity, truth

\section{Introduction}

"Beauty is truth, truth is beauty", the closing line of John Keats's Ode on a Grecian Urn, draws attention not only of literary critics but also of scientists who often quote it when reflecting on the beauty they see in nature. In particular, many physicists give a lot of credit to beauty in natural laws, up to the point that beauty is as important for them (as scientists) as truth. As we will see, for a few it can be even more important than truth. This might be a bit unexpected - after all, the job of a physicist is to look for truth, not beauty. And some scientists, afraid that unlimited fascination with beauty can lead us astray, would agree. Ken Lane once wrote that when in search for truth, "leave beauty to tailors and bootmakers [1]".

Jesus Zamora Bonilla classifies the main positions about this question in the following way [2]: "In the first place, there are philosophers and scientists that insist on the claim that the pursuit of beauty is an important, even an essential ingredient in the process of scientific research. In the second place, there are those that simply deny that scientific research has anything intrinsic to do with beauty. ... it is just a coincidence that some empirically successful theories trigger something like a sense of beauty." Bonilla refers to the former as 'the Platonists' since their view is usually associated with the idea that the ultimate explanation of the universe must possess beauty. The latter group he calls 'Sceptics' and claims this position is "the most common within the philosophers of science, and I would venture to say within most practicing scientists outside the most abstruse fields of quantum physics and higher mathematics". He also labels Richard Feynman, the Nobel Prize-winning theoretical physicist, as Sceptic, quoting his famous line "it doesn't matter how beautiful your theory is, it doesn't matter how smart you are: if it doesn't agree with experiment, it's wrong".

In this contribution, as a scientist representing one of those most abstruse fields (particle physics), I will look at views of physicists who valued beauty dearly, give criteria for what they mean/meant by beauty followed by examples of beautiful physical laws, and finally take a look at some dissenting opinions.

\section{Proponents of beauty}

Many physicists of the 19th and 20th centuries appreciated the importance of aesthetic values in their theories. Henri Poincare, a French mathematician and theoretical physicist, once said "The Scientist does not study nature because it is useful to do so. He studies it because he takes pleasure in it; and he takes pleasure in it because it is beautiful" [3]. J.W.N. Sullivan, a British physicist and science writer, argues "The measure of the success of a scientific theory is, in fact, a measure of its aesthetic value, since it is a measure of the extent to which it has introduced harmony in what was before chaos" [3]. Albert Einstein described Niels Bohr's atomic model as the highest form of musicality in the sphere of thought [4].

Heinrich Hertz, who proved the existence of the electromagnetic waves, wrote about his feeling that James Clerk Maxwell's fundamental equations of electricity and magnetism "have an independent existence and an intelligence of their own, that they are wiser ... even than their discoverers, that we get more out of them than was originally put into them" [4]. Even Richard Feynman, quoted as Sceptic by Bonilla, said "You can recognize truth by its beauty and simplicity. When you get it right,

\footnotetext{
* Ivan Melo

Department of Physics, Faculty of Electrical Engineering, University of Zilina, Slovakia

E-mail: melo@fyzika.uniza.sk
} 
it is obvious that it is right - at least if you have any experience because usually what happens is that more comes out than goes in" [5]. What Hertz and Feynman refer to, became a criterion for a beautiful theory: more comes out than goes in (see Sec. 3). More recently, the 2004 Nobel prize laureate Frank Wilczek expressed his view in this way "My work has been guided by trying to make the laws more beautiful" [6].

Reading these statements, one can feel that for their authors beauty was likely as important as truth. But there is another group of physicists who seem to go even further. Hermann Weyl, a German mathematician, physicist and philosopher made a surprising claim: "In my work, I have always tried to unite the true with the beautiful; but when I had to choose one or the other, I usually chose the beautiful" [3]. Murray Gell-Mann, an American physicist who received the 1969 Nobel Prize for his work on the theory of elementary particles, would agree: "Beauty is a very successful criterion for selecting the right theory... In 1957 we published a partial theory of weak interactions which disagreed with seven experiments. We believed they were wrong because our theory was beautiful ... and they were" [7].

Albert Einstein was so convinced about the beauty of his theory of special relativity that when asked about the experiment (by D.C. Miller) which appeared to disagree with it, he responded

"Oh, that will go away" [7]. In 1919, when Arthur Eddington, an English astronomer, put general relativity to a successful test, Einstein was asked what if the results hadn't agreed with the theory? His response was "I would have been sorry for the dear Lord, for the theory is correct" [8].

We conclude this list with the Nobel Prize-winning physicist Paul Dirac, who said in 1963 that "it is more important to have beauty in one's equations than to have them fit experiment" [8].

\section{Beauty criteria}

What kind of beauty are all these great men talking about? Beauty can be very subjective as our experience with works of art tells us. Subrahmanyan Chandrasekhar, an Indian astrophysicist famous for his work on the evolution of stars, suggests two criteria for the beauty of a physical law [3].

The first is due to Francis Bacon: There is no excellent beauty that hath not some strangeness in the proportion! The second was formulated by Werner Heisenberg, a German theoretical physicist and one of the key pioneers of quantum mechanics: Beauty is the proper conformity of the parts to one another and to the whole.

I think I understand these criteria, but they seem a little vague to me. I prefer the two criteria suggested by Frank Wilczek and others [6] which find a broader acceptance among physicists. Wilczek calls the first one productivity, getting out more than you put in: "You find some equation or law by putting together clues and making a guess, and then you can explain seven other things and you know you're on the right track. You get out more than you put in." I agree. If, starting from a single equation, you can predict many diverse phenomena, it seems obvious that there is power and simplicity in the equation that Feynman and Hertz talked about. The more phenomena one can explain with fewer equations, the more beautiful the theory is.

The second criterion is symmetry. Symmetry is defined in an objective way in physics. As Wilczek puts it, it's change without change. "You can make changes in physical objects or changes in the laws that could change them but don't" [6].

The symmetry proved to be especially fruitful in fundamental physics. Let us give some examples. If we rotate a circle by any angle around its center, there is no visible change. We did something, but the effect is none. The circle is a very symmetric object. Early astronomers, likely with this symmetry in mind, assumed that planetary orbits were perfect circles. As Johannes Kepler painstakingly discovered, this was wrong. The orbits are elliptical. Sometime later Isaac Newton formulated the theory of gravity that explained why orbits actually trace ellipses. It turned out that his theory has a circle symmetry - the gravitational force between two objects is the same if we rotate one object around the other by any angle. We made a change, but the law did not change. Here we have an important lesson. Our intuition about the importance of symmetry was correct, but the symmetry did not apply to the orbits (the solutions of the gravitational law), but to the law itself. This is lovely according to a physicist taste. There can be many solutions to the law, some beautiful, some less so, but the law behind is one, and it is beautiful.

The circle symmetry is just the simplest example of symmetry discovered in nature. Einstein built his special theory of relativity on a postulate that the laws of physics are the same in the so-called inertial frames of reference. That is, the laws of physics will not change under Lorentz transformations which include both spacial rotations and transformations to the frames which move with constant speed with each other (we say that the laws have Lorentz symmetry). The Maxwell's equations of electricity and magnetism are an example of a law which does not change under Lorentz transformations. We can see that different symmetries operate in this world and physicists are busy in an effort to uncover them all. The Maxwell's equations also illustrate the first criterion at work: from just four equations one derives all electricity, magnetism, and optics.

The Maxwell's equations have one other symmetry, the gauge symmetry, which had a profound effect on our understanding of the Standard Model of particle physics.

In Quantum electrodynamics (QED), the quantum version of Maxwell's theory and part of the Standard model, the gauge symmetry is the principle from which everything follows.

Curiously, the QED gauge symmetry is a circle symmetry but with a new important twist: we take the field which represents the electron at some point in spacetime and rotate it by a certain 
angle ${ }^{1}$ and do this at each point in spacetime with a different angle of rotation. If we now demand that the energy of the electron field does not change under this gauge transformation, a miracle happens: one is forced to introduce the electromagnetic field which interacts with the electron field precisely as described by Maxwell's equations.

We started with basically nothing but the electron field and demanded the gauge symmetry (and the Lorentz symmetry to be precise), in this particular case the circle symmetry. The nontrivial thing was to rotate the field in a circle by different angles for different spacetime points (by contrast, in the case of Newton's theory of gravity above, we had only one angle for all space). From this minimal assumption, the existence of electromagnetic interactions follows and Maxwell's equations are derived.

A similar approach was successfully applied also to weak and strong interactions. The symmetries, in this case, are more complicated than the circle symmetry but the gauge principle is there, and its beauty is even more profound. The Standard model is the gauge theory of the electromagnetic, weak and strong interactions. Gravitational interactions are not included in this model yet. Hermann Weyl formulated the gauge theory of gravity long ago, then realized it does not describe reality. However, he liked it so much that he made his famous statement regarding the relation between beauty and truth, see Sec. [2].

I admit that this view of beauty is likely different from what we find in arts. Philip Ball makes important observations in [8], quoting the philosopher of chemistry Joachim Schummer and the art historian Ernst Gombrich. Schummer pointed out that by redefining beauty as symmetry one deviates from the dominant tradition in artistic theory. "There's a reason why our galleries are not, on the whole, filled with paintings of perfect spheres". Gombrich said that too much symmetry ensures that "once we have grasped the principle of order ... it holds no more surprise". Artistic beauty, Gombrich believed, relies on a tension between symmetry and asymmetry. Ball adds "the field of physics has always been heir to Platonism - the mystical conviction of an orderly cosmos. ... for Plato it was precisely art's lack of symmetry (and thus intelligibility) that denied it access to real beauty. Art was just too messy to be beautiful."

In conclusion of this section, I would like to make a note. Beauty is often viewed by philosophers as simplicity, and this category can indeed be found in both of the Wilczek's criteria. If we can explain many phenomena with a single equation, the theory is not only productive; it is also simple, certainly simpler than the theory which needs many equations to accomplish the same thing. If we move to the second criterion, the symmetric objects or theories are, as Gombrich puts it, easy to grasp and

${ }^{1}$ The electron field is a complex function with real and imaginary parts and the rotation takes place in this complex plane, not in spacetime. 'easy to understand' is a synonym for simple (simplicity, however, can be realized in a tricky way, as our example with the simple circle symmetry in QED shows).

\section{Skeptics?}

Modern particle physicists are in general also proponents of beauty. It is hard to deny that fundamental laws are beautiful and this became motivation for many of us to do physics.

There is, however, a point I would like to make: the emphasis some of the great minds of the 19th and 20th centuries put on beauty, especially if they prefer it to truth, may seem far-fetched for our generation. In my training as a physicist, we were taught (and rightly so) that experiment, not beauty, is the judge between competing theories. As Brian Greene says in the Elegant Universe: "Ultimately, theories are judged by how they fare when faced with cold, hard, experimental facts." According to Greene, Einstein (and others) was just saying that beauty in a theory is a good guide, an indication that you are on the right track [8].

I am not sure if this was Einstein's and Weyl's point, but I admit it sounds a reasonable position. We may have two beautiful theories but only one of them (or none) can be true, and the experiment is the final arbiter. The role of beauty as a guide is the role of a good servant of the truth. We prefer a beautiful theory to an ugly one with pragmatic motives in mind.

Wilczek made an important point along these lines: "Today's frontiers of fundamental physics are far removed from everyday experience. They are difficult and expensive to access experimentally, and we cannot rely on our intuition to fill in the blanks. The patient accumulation of fact recommended by Francis Bacon, Isaac Newton, and Sherlock Holmes is thus no longer practical. Instead, we reverse the process, using guesswork to motivate experiment. We first construct beautiful equations, then derive their consequences, and, finally, craft experiments to test them. In recent decades, that strategy has proved remarkably successful... Rather than recognizing the beauty of laws otherwise discovered, we use principles of beauty - vast symmetry and a high ratio of output to input - to enable discovery" [4].

As Wilczek says, this approach was extremely useful in building modern quantum theories which culminated in the construction of the Standard Model of elementary particles. We might say that without giving beauty a very high priority, there would likely be no Standard Model, no Large Hadron Collider (LHC) and no Higgs boson discovered. And physicists continue in this direction. The LHC was motivated not only by the Standard Model and the Higgs boson but also by new theories which go beyond the Standard Model. These new theories were built with aesthetic arguments as their cornerstones.

For example, supersymmetric theories represent the only nontrivial way to extend the spacetime symmetries such as Lorentz symmetry (gauge symmetries and other internal symmetries can 
be combined with spacetime symmetries only trivially). This is a pure mathematical argument, powerful and beautiful at the same time. But there are also two physical arguments with a high aesthetic value in favour of supersymmetry: supersymmetry, unlike the Standard Model, offers a way to unify the three interactions, electromagnetic, weak and strong into a single one and at the same time it provides a good dark matter candidate - the lightest among the many new supersymmetric particles predicted by the theory. Naturally, the search for supersymmetry has become a key part of the LHC physics programme. The expectation is that at least some of the new particles should be visible at the LHC.

And here a possible rift in the seemingly homogeneous views of particle physicists towards beauty is looming. With five years after the Higgs boson discovery, we have not seen any new fundamental particle. Neither supersymmetric, nor other. Much to our disappointment, the Standard Model passes all the experimental tests with flying colours.

Reactions to this differ. Some keep their belief in supersymmetry arguing that the LHC has so far collected only a few percents of the designed total amount of data. Only the simplest versions of supersymmmetry, they say, are in difficulties when confronted with facts. The more sophisticated forms of the theory allow more space where the new particles can be hiding.

Others are beginning to have doubts. They are concerned that to continue the hunt for new particles just because the theory which predicts them is aesthetically pleasing, is not the best way to proceed. Beauty, they think, should not count as indirect evidence in favor of an idea [9]. Sarah Demers, a professor at Yale University, thinks that maybe it is okay for the universe to be a little bit ugly and Marcelo Gleiser, a professor of physics at Dartmouth College adds "... perhaps the insistence that we have in search of perfect symmetry is not a physics idea, but a bias [9]." The way out, according to Demers, is to let data lead the way. "I think we may be more likely to win by the data just forcing us in a direction, as opposed to having some great idea that's aesthetically motivated," she says. Physicists can still search for evidence of the Standard Model and supersymmetry, but they should also go on "agnostic quests," Demers says, where they don't go looking for something in particular.

Up to this point, the arguments seem justified. If beauty fails to help us find truth, it makes perfect sense to look into data instead without any bias and see what we find. This is exactly what Kepler had done and found that planetary orbits were elliptical. Of course, the question is if the time has come. Right now at the LHC both unbiased and biased searches are being performed.

Gleiser, however, takes another step and in pursuit of further unification, he sees a renewed religious impulse. "The idea that there is a force that describes everything is sort of a monotheistic cultural vice that we have," he says. "Growing up in a culture for two or three thousand years where there is a god and a central command of things - I think that's deeply ingrained in people's heads." In some sense, physicists have replaced their one true, symmetrically-faced God with one true, symmetric theory [9].

Here, a group of Sceptics is coming out. Too much flirting with beauty, they think, has religious connotations. Or, as another group claims, there is nothing special about beautiful laws - they seem beautiful to us because we evolved and grew up with them and therefore are used to them. Somehow, both groups deny beauty its special status, its objective existence.

I think that "Platonists" need not worry. Their conviction is, after all, based on facts and previous experience. The Standard Model and General relativity describe reality very well and are beautiful. It is very natural to assume that the same is true also for theories which go beyond the Standard Model. One does not have to believe in God to do so. Of course, if the facts point (just point, not prove) to universal beauty, order, harmony, and unification, to a believer these attributes could sound familiar.

\section{Conclusions}

Beauty can be defined in quite an objective way using productivity and symmetry as criteria.

Many particle physicists are truly fascinated with beauty, many others view it more pragmatically, and a few others are ready to challenge its high priority seat if an opportunity occurs.

But all would likely agree that beauty has been an essential ingredient in the process of scientific research in our field so far. Even if there is no "theory of everything" that unites all laws of physics, Frank Wilczek says "we already know there are beautiful laws that explain most of the way matter works. It's just that we haven't figured it all out. It's difficult to exaggerate how symmetric, how fruitful, how creative the laws are. It's a great gift" [6]. I conclude that in spite of slightly different attitudes among physicists, beauty is not just for tailors and bootmakers.

\section{Acknowledgement}

I would like to thank the members of the International Particle Physics Outreach Group [10], in particular Hans Peter Beck, Dezso Horvath, Steven Goldfarb, Peter Watkins, Thomas Naumann, Alberto Ruiz Jimeno, Miguel Sanchis and Achintya Rao for their interest and stimulating discussions of this subject. 


\section{References}

[1] LANE, K.: Invited Talk Given at the International Conference on the History of Original Ideas and Basic Discoveries in Particle Physics, Sicily, 1994 [online]. Available: https://arxiv.org/abs/hep-ph/9501249v1.

[2] BONILLA, J. Z.: Science and the Search of Beauty [online]. Mapping Ignorance, 2015. Available: https://mappingignorance. org/2015/01/28/science-search-beauty-1/.

[3] CHANDRASEKHAR, S.: In his talk "Beauty and the Quest for Beauty in Science" given in the Fermilab Symposium, Aesthetics and Science [online]. 1979. Available: http://physicstoday.scitation.org/doi/abs/10.1063/1.3529003?journalCode=pto.

[4] Why Is Physics Beautiful? [online]. Project Syndicate, 2015. Available: https://www.project-syndicate.org/commentary/why-isphysics-beautiful-by-frank-wilczek-2015-10.

[5] FEYNMAN, R., attributed, Sympathetic Vibrations. See also http://www.azquotes.com/quote/1091754.

[6] PAULSON, S.: Beauty Is Physics' Secret Weapon [online]. 2016. Available: http://nautil.us/issue/32/space/beauty-is-physicssecret-weapon.

[7] GELL-MANN, M.: TED Talk Beauty and Truth in Physics [online]. Available: https://www.ted.com/talks/.

[8] BALL, P. [online]. Available: https://aeon.co/essays/beauty-is-truth-there-s-a-false-equation.

[9] SCOLES, S.: What Does Beauty Have To Do with Physics? [online]. Nova Next, 2016.Available: http://www.pbs.org/wgbh/nova/ next/physics/beauty-in-physics/.

[10] International Particle Physics Outreach Group [online]. Available: http://ippog.org/. 